\title{
Electron-hole correlation effects in the emission of light from quantum wires
}

\author{
Francesco Tassone \\ Ginzton Laboratory, Stanford University, Stanford, CA-94305, and ERATO Quantum Fluctuation Project \\ Carlo Piermarocchi \\ Institut de Physique Théorique, Ecole Polytechnique Fédérale, CH-1015 Lausanne, Switzerland
}

(May 11, 2018)

\begin{abstract}
We present a self-consistent treatment of the electron-hole correlations in optically excited quantum wires within the ladder approximation, and using a contact potential interaction. The limitations of the ladder approximation to the excitonic low-density region are largely overcome by the introduction of higher order correlations through self consistency. We show relevance of these correlations in the low-temperature emission, even for high density relevant in lasing, when large gain replaces excitonic absorption.
\end{abstract}

Based on the singularity of the density of states (DOS) of massive particles at the band edge in one dimension (1D) and the single particle picture, large gains and significant improvements in optical devices have been predicted for 1D systems. 咽 However, inclusion of the Coulomb correlations among the photoexcited carriers results in a finite absorption at the band gap, i.e. in a vanishing Sommerfeld factor. the large exciton binding energy and oscillator strength, which strongly reduces that available for free carriers. Correlation between electrons and holes is therefore very important in 1D, and likely to persist up to large carrier densities. It has been shown that screening in $1 \mathrm{D}$ systems has a little influence on optical nonlinearities, 1 and in a first approximation can be neglected. Thus, a minimal model describing the optical properties of the wires has to include the strong Coulomb correlation between electron and holes, both in the low density excitonic limit and for the higher densities, when the Fermi gas becomes degenerate. Therefore, the time dependent Hartree-Fock (HF) approximation for the electron-hole system, which is used in the derivation of the semiconductor Bloch equations, is not sufficient for this purpose. Indeed, this fact is also recognized for higher dimensional systems, where, beside screening effects, the time-dependent HF was improved by including scattering between carriers at the Born level 9 But, even if in the optical response the exciton resonance is reproduced, the excited electrons and holes in this model are still the single particle states of the Hartree-Fock theory, thus bound electron-hole pairs are not included. In this work we go beyond these approximations, and include correlation effects by summing up electron-hole scattering to infinite order in laddertype diagrams (ladder approximation, LA). The resulting renormalized interaction (T-matrix) is known to correctly reproduce the bound states at low carrier densities, and Coulomb correlations among the unbound states Phase-space filling effects are also partially included.I However, the range of validity of the LA is in fact rather limited, as higher order correlations become important at higher densities. These can be partially recovered within a self-consistent approach (self consistent ladder approximation, SCLA), where the bare electron and hole propagators are dressed to include the effects of scattering with other carriers on propagation. In this way, multiple scattering between bound and unbound pairs are effectively included $\mathbf{3}$ These correlations are relevant for temperatures smaller than the exciton binding energy, which is significantly large in these systems. In this work we consider a simplified coulomb interaction potential in order to clearly assess this statement and compare its results to simpler models, which do not include correlation. As an interesting example, we finally show that emission calculated within our model shous qualitative agreement with experimental observations, explaining the origin of apparent absence of band gap renormalization in the emission for the wires.

We here consider one parabolic, and spinless, conduction band for electrons (creation operators $c_{k}$ ) and one for holes $\left(d_{k}\right)$, with the same masses, interacting through a simple contact potential $V(x)=a \delta(x)$, where $a=2 E_{b} a_{B}, E_{b}$ and $a_{B}$ being the binding energy and the Bohr radius of the exciton. Electrons and holes are independent fermions, and the corresponding operators commute. We introduce dimensionless units, where $E_{b}=1 / 2$, and lengths are scaled with the $1 \mathrm{D}$ Bohr radius. Then, the Hamiltonian reads

$$
\mathcal{H}=\sum_{k} \frac{k^{2}}{4}\left[c_{k}^{\dagger} c_{k}+d_{k}^{\dagger} d_{k}\right]-\sum_{k, k^{\prime}, q} c_{k-q}^{\dagger} d_{k^{\prime}+q}^{\dagger} d_{k^{\prime}} c_{k}
$$

The choice of the contact potential makes T-matrix selfconsistency manageable to both numerical and analytical treatment. Within this potential, electron-electron and hole-hole interactions are absent for the same spin. Although a drawback for realistic calculations, it allows us to focus on the electron-hole correlation alone. We also remark that screening can not be consistently introduced within such a restricted form of the potential. But this is presumably a minor drawback in $1 \mathrm{D}$, as remarked above. Standard finite temperature Green function techniques may be used to write the SCLA. However, the solution 
of the self-consistent problem is by necessity numerical, and the analytical extension to real frequencies is numerically difficult, due to the complex structure of the resulting Green functions 10 For this reason we work directly with real frequencies/times, where the Green functions and self-energies are matrices defined on the Keldysh contour, 11 and their matrix elements related by the Kubo-Martin-Schwinger relations for thermal equilibrium. The full SCLA reads:

$$
\begin{gathered}
T_{k}(\omega)=1+\int_{C} d \omega^{\prime} \sum_{q^{\prime}}\left[i G_{k-q^{\prime}}\left(\omega^{\prime}\right) G_{q^{\prime}}\left(\omega-\omega^{\prime}\right)\right] T_{k}(\omega) \\
G_{k}(\omega)=G_{k}^{(0)}(\omega)+G_{k}^{(0)}(\omega) \Sigma_{k}(\omega) G_{k}(\omega) \\
\Sigma_{k}(\tau)=\sum_{q} i T_{k}(\tau) G_{q-k}(-\tau)
\end{gathered}
$$

The SCLA is a conserving approximation 2 : particle number and energy are conserved, making the SCLA robust at any density, even when large scattering produce significant renormalizations. A detailed study of SCLA for both the attractive and repulsive (single-band) Hubbard model at half filling shows that the SCLA is substantially close to the available exact solutipn in an extensive range of temperatures/magnetizations. 13 We expect this to be even more true for this case of negligible fillings (continuum model).

In the low density limit, using the bare particle propagators, the $T$-matrix shows a pole at the exciton energy, $T^{+}(k, \omega) \simeq\left[\omega-\left(-1 / 2+k^{2} / 8\right)+2 i \gamma\right]^{-1}$. At the next selfconsistency step, the particle propagators show structures below the exciton energy through the self-energy $\Sigma$. Higher order terms in self-consistency then introduce exciton-exciton scattering effects. 8 These effects are actually overestimated for the contact potential with respect to a realistic coulomb potential due to the lack of electron-electron (and hole-hole) interactions in the spinless model 14 We also checked that inclusion of spin in the SCLA (using the contact potential) reduces scattering for a given density. In order to visualize the effects of self-consistency on the single particle Green function, we plot in Fig. 1 the spectral function at different steps of iteration for $T=0.1$. At step 0 , the spectral function shows the usual single particle single peak, broadened by $\gamma=0.02$ for regularization. At step 1, excitonic correlations appear. Due to finite temperature, not only a single excitonic peak appears, but contributions from larger exciton momenta are also evident. The discretization of the $\mathrm{k}$-space (16 points) also results in a fragmentation of this two particle continuum into resolved peaks, and in a reduction of the binding energy at low densities from 0.5 to 0.37. Further self-consistency steps significantly broaden and shift both the single particle and two-particle peaks. We thus understand that interaction among carriers is shifting both exciton energies and single particle energies.

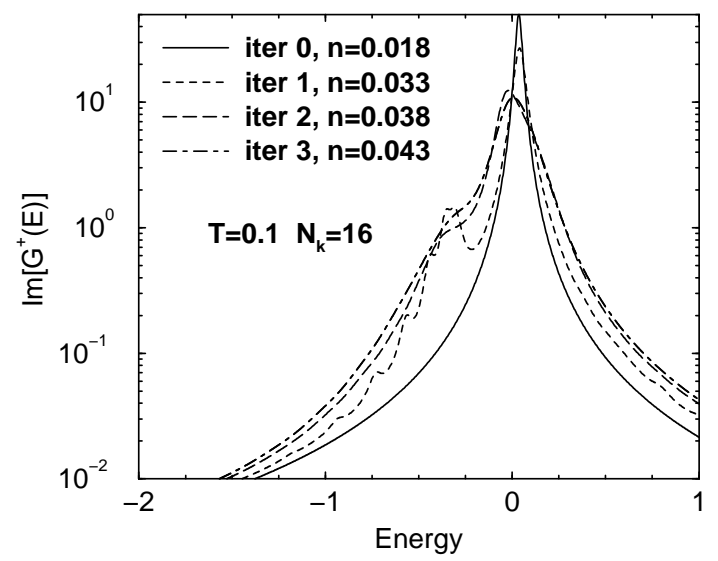

FIG. 1. The spectral function $\Im\left[G^{+}\right]$at $\mathrm{k}=0$, first four steps of iteration. Corresponding densities are also shown. Other parameters in the figure.

Next, we introduce the optically measurable quantities, emission (or photoluminescence), and absorption. We calculate them in the dipole approximation, and neglecting retardation in the electromagnetic interaction between carriers (polaritonic effects). The polarization $P$ is the linear response to the classical external transverse field, calculated within the dipole approximation. It is thus related to the two-particle electron-hole Green function, where the external electron and hole lines are closed over the interaction with the transverse photon:

$$
\begin{gathered}
P(\mathbf{k}) \propto \sum_{\mathbf{q}, \mathbf{Q}} G_{2}\left(\frac{\mathbf{k}}{\mathbf{2}}-\mathbf{q}, \frac{\mathbf{k}}{\mathbf{2}}+\mathbf{q} ; \frac{\mathbf{k}}{\mathbf{2}}-\mathbf{q}-\mathbf{Q}, \frac{\mathbf{k}}{\mathbf{2}}+\mathbf{q}+\mathbf{Q}\right)= \\
H(\mathbf{k})+H(\mathbf{k}) T(\mathbf{k}) H(\mathbf{k}),
\end{gathered}
$$

with $H(\mathbf{k})=i \sum_{\mathbf{q}} G(\mathbf{k} / 2-\mathbf{q}) G(\mathbf{k} / 2+\mathbf{q})$. Here we used the notation $\mathbf{k}=(k, \omega)$, and optical frequencies are measured from the gap. The first term describes the usual band to band recombination found in the single particle picture, whereas the other term includes the correlation effects of bound electron-hole states. Here we notice that Eq. (2) gives the linear response of the system to the external field only when the exact $G_{2}$ is used 12 However, we may expect that it is accurate also in the SCLA as we noticed above that the SCLA is expected to be quantitatively close to the exact solution. We checked that deviations from current-conservation in the linear response are negligibly small.

The absorption $\alpha$, related to $\Im\left[P^{+}\right]$, (the + apex labelling the retarded function), can be written as

$$
\alpha(\mathbf{k}) \propto \Im\left[H^{+}(\mathbf{k})\right]\left|T^{+}(\mathbf{k})\right|^{2} .
$$

Thus, absorption in the excitonic limit shows a pole at the exciton energy at low densities. Moreover, we may also analytically find the absorption above the gap by using the analytic expression of $H^{+}(k=0, \omega)=$ $-i / \sqrt{2 \omega+i 0^{+}}$for small densities:

$$
\alpha(k=0, \omega)=\frac{\sqrt{2 \omega}}{1+2 \omega}
$$


which clearly shows cancellation of the 1D DOS divergence at $\omega \rightarrow 0^{+}$, i.e. a Sommerfeld factor which vanishes linearly at $\omega=0$. The $k=0$ corresponds to the direction perpendicular to the wire. Emission is instead related to the correlation function of the dipole operator and therefore to $P^{<}(k, \omega)$. It can be found from absorption through the Kubo Martin Schwinger relations:

$$
P^{<}(k, \omega) \propto[\exp \beta(\omega-2 \mu)-1]^{-1} \Im\left[P^{+}(k, \omega)\right]
$$

which show the typical Bose occupation factor for the emission. We conclude that at low densities/temperatures, emission is excitonic as described in absorption by the poles of $T^{+}$.

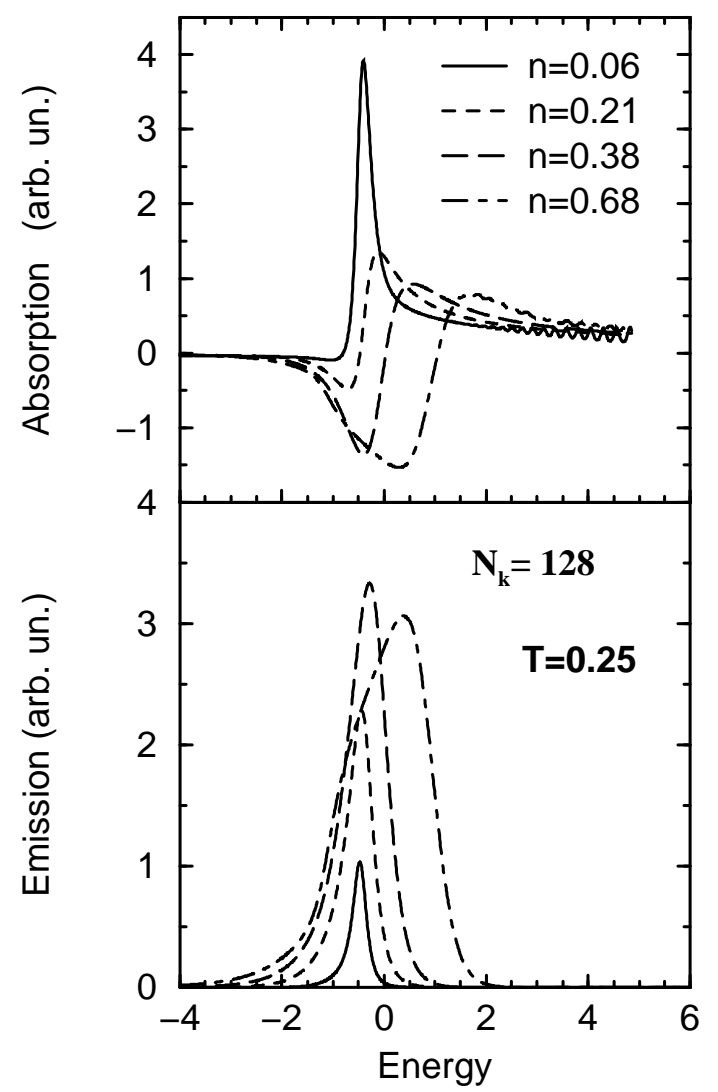

FIG. 2. The absorption and emission at various densities. Other parameters in the figure.

In the following, we discuss emission and absorption at intermediate and high densities, when a relevant number of unbound pairs coexist with bound pairs. This situation is relevant for the photoluminescence experiments, and for low temperature laser operation, when hot carriers are injected in the system. Of course, quite stronger correlation effects can be found at smaller temperatures, when most of the carriers are bound into excitons, but our aim is to show the relevance of correlation in a typical and less extreme situation. We solved Eq. (何) for $\mathrm{T}=0.25$ (corresponding to $E_{b} / 2$ ) and densities up to $\mathrm{n}=0.8$. In Fig. 2 we plot the resulting absorption and emission. The low density limit shows the excitonic absorption, and the band to band absorption, as a long tail at higher energy. At larger densities, excitonic absorption is bleached and gain becomes evident. Even for $\mathrm{n}=0.38$, when large gain is present in the exciton spectral region, the emission peak stays fixed close to $E_{b} \sim-1 / 2$, the low-density exciton energy. At the largest densities $\mathrm{n}>0.4$, the emission intensity saturates and a flattened top of the emission peak results from the significant depth of the Fermi sea of electrons and holes. We notice that even for $\mathrm{n}<0.4$, significant broadenings are predicted by the SCLA. These are instead absent in the HF calculations and have to be introduced either phenomenologically, or by treating scattering among carriers at the Born level. We remark that scattering at the Born level is just the first of the infinite terms in the ladder expansion. In order to better understand the origin of the stability of the energy of the emission peak with density, we compared the exciton energy, the emission energy, and the band gap renormalization (BGR), defined as twice the shift of the single particle energy (Fig. 3). This definition coincides with the optical band gap renormalization at small densities. We also defined the exciton energy as that of the pole of the T-matrix (more precisely, the $\left|T^{+}\right|^{2}$ peak).

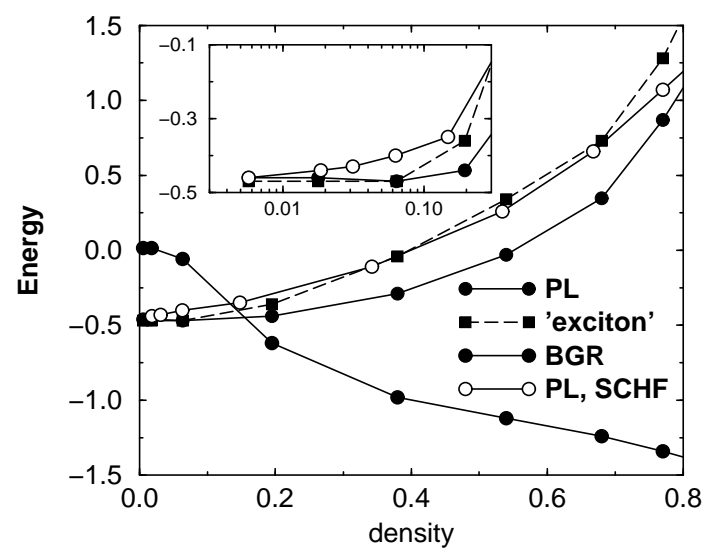

FIG. 3. Emission peak energy (PL), emission peak energy in HF (PL-SCHF), the exciton energy $\left(\left|T^{+}\right|^{2}\right.$ peak), and band gap renormalization (BGR) as functions of the density. In the inset an enlargement for the lower densities.

In Fig. 3 we notice large negative BGR even at relatively small density. For $n \sim 0.15$, the BGR is comparable to $E_{b}$, but a small change in the exciton energy is observed (Fig. 3, inset). We remark that the values of the BGR are comparable to those calculated using a more realistic cowomb potential within the random phase approximation 15 It confirms that screening effects on BGR are small for low densities, when the depth of the Fermi sea is smaller than the temperature as in our case. Intuitively, in our model BGR and binding energy compensate to produce a negligible shift of the exciton energy. However, this compensation goes well beyond the densities where the concept of exciton as a bound state is still relevant, i.e. when the bandgap falls below the exciton energy, at $n>0.15$. Here, the T-matrix 
still shows a peak. It is now originating from a twoparticle scattering resonance in the two-particle continuum. From Fig. 3, we notice an agreement between the exciton energy and emission (PL) peak for $n<0.2$. It' $\mathrm{s}$ remarkable that the emission peak does not shift much even for $0.2<n<0.4$, when the exciton energy does. But, this discrepancy between the two appears in a region where significant broadening of both the peaks is present, as clearly shown in Fig. 2 for the emission. This stability of the emission peak is not correctly predicted by the simpler self-consistent $\mathrm{HF}$ approximation (using the same contact potential for consistency), also shown in Fig. 3. Even at lower densities (Fig. 3, inset), when self-consistent HF correctly predicts excitonic emission, larger emission shifts are found compared to the SCLA. Moreover, we again remind that the HF approximation does not consistently introduce broadenings in the spectra. Only at densities $n>0.8$ the self-consistent $\mathrm{HF}$ and the SCLA coincide, given the larger weight of uncorrelated carriers in the system at these large densities. We here remark that the same fixed emission peak for a broad range of densities was also observed in recent experiments on high quality GaAs quantum wires at comparable temperatures, and attributed to excitonic emission 9 From this emission data, the authors also concluded that BGR is negligible. Here, we show instead that emission data is not sufficient to support this conclusion, and that indeed BGR can coexist with a fixed emission peak in the $1 \mathrm{D}$ system. We also remind that our simplified model is overestimating broadenings and shifts, so that this conclusion holds even more true.

In conclusion, we have shown the importance of electron-hole correlation effects in the emission of light from quantum wires, even at large densities, when the temperature is smaller than the exciton binding energy. We discussed how the self-consistent ladder approximation includes a large part of these correlations, and in particular the broadening and renormalization resulting from multiple scattering between correlated pairs. It is able to predict negligible shifts of the emission peak in a large range of densities, in agreement with experimental observations, and in contrast to the predictions of simpler Hartree-Fock approximation. Moreover, we observed that this feature coexists with significant band-gap renormalization, and even with the disappearance of the excitonic peak in absorption. The SCLA is thus the simplest model retaining sufficient correlations to explain gain and emission characteristics of the wires at low temperatures, even in the high-density regime relevant for lasing.

We thank R. Ambigapathy, C. Ciuti, B. Deveaud, A. Quattropani, V. Savona, P. Schwendimann, P. E. Selbmann, Y. Yamamoto, and R. Zimmermann, for many stimulating discussions. The present work has been supported by the Swiss National Science Fundation, by the Swiss National Priority Program for Optics, and by the ERATO Quantum Fluctuation Project.
${ }^{1}$ Y. Arakawa and H. Sakaki, Appl. Phys. Lett. 40, 939, (1982).

${ }^{2}$ E. Kapon, Proc. IEEE 80, 398, (1992).

${ }^{3}$ T. Ogawa and T. Takagahara, Phys. Rev. B 43, 14325, (1991).

${ }^{4}$ F. Rossi and E. Molinari, Phys. Rev. Lett. 79, 3642, (1996).

${ }^{5}$ S. Benner, and H. Haug, Europhys. Lett. 16, 579 (1991).

${ }^{6}$ M. Lindberg and S.W. Koch, Phys. Rev. B 38, 3342, (1988).

${ }^{7}$ for a review see e.g., R. Zimmermann, Many Particle Theory of Highly Excited Semiconductors, (BSB Teubner, Liepzig, 1987).

${ }^{8}$ R. Haussman, Z. Phys. B 91, 291, (1993).

${ }^{9}$ R. Ambigapathy et al., Phys. Rev. Lett. 78, 3579, (1997).

10 S. R. White et al., Phys. Rev. Lett. 63, 1523, (1989).

${ }^{11}$ P. Danielewicz, Annals of Physics 152, 239, (1984).

12 G. Baym and L. P. Kadanoff, Phys. Rev. 124, 287, (1961).

${ }^{13}$ F. Buzatu, Modern Physics Letters B 9, 1149, (1995).

${ }^{14} \mathrm{~F}$. Tassone and C. Piermarocchi, unpublished.

15 Ben Yu-Kang, and S. Das Sarma, Phys. Rev. B 48, 5469, (1993). 